\title{
The Influence of Age and Gender in the Interaction with Touch Screens
}

\author{
Rodrigo Rocha ${ }^{1}$, Davide Carneiro ${ }^{1,2}$, and Paulo Novais ${ }^{2}$ \\ 1 CIICESI/ESTG - Polytechnic Institute of Porto \\ Felgueiras, Portugal \\ \{8140411, dcarneiro\}@estg.ipp.pt \\ 2 Algoritmi Centre/Department of Informatics, Universidade do Minho, Portugal \\ pjon@di.uminho.pt
}

\begin{abstract}
Touch screens are nowadays one of the major interfaces in the interaction between humans and technology, mostly due to the significant growth in the use of smartphones and tablets in the last years. This broad use, that reaches people from all strata of society, makes touch screens a relevant tool to study the mechanisms that influence the way we interact with electronic devices. In this paper we collect data regarding the interaction patterns of different users with mobile devices. We present a way to formalize these interaction patterns and analyze how aspects such as age and gender influence them. The results of this research may be relevant for developing mobile applications that identify and adapt to the users or their characteristics, including impairments in fine motor skills or in cognitive function.
\end{abstract}

Keywords: Human Computer Interaction, Touch Screens, Human Factors

\section{Introduction}

In the last years the use of devices such as smartphones and tablets has grown immensely [1]. Due to this, the touch screen emerges as one of the most used interfaces for human-computer interaction and, the gesture of the touch, as the most common form of interaction. When compared to other forms of interaction, such as the now more traditional mouse and keyboard, the touch screen is a much more intuitive one, which in part accounts for the acceptance and use of tactile devices by so many different strata of society, with so many different socio-economic contexts.

These means of interaction are interesting in the sense that they allow to collect information that may characterize the user of the device or her/his state. The underlying principle is that, much like in our inter-personal interactions, aspects such as our emotions, our surroundings, and many others, affect our interaction. Specifically, they do not affect so much the content of our interaction (i.e. the words spoken) but rather the way we speak them [2]. This is why we are able to perceive if our interlocutor is stressed, tired or experiencing some specific 
emotion without explicit information: we do so by (sometimes subconsciously) analysing the tone of the voice, the intonation or the posture of our interlocutor.

Recent findings show that our interaction with technological devices such as the keyboard and the mouse is modulated in a similar fashion. Our research team demonstrated how stress and mental fatigue affects one's interaction with the computer $[3,4]$.

Other researchers have also studied related topics, such as the influence of age and gender on keystroke dynamics (one's typing patterns) and mouse dynamics (one's interaction patterns with the mouse) [5] or the influence of emotion on the same features as well as on text production [6].

Some researchers also started studying similar phenomena on the interaction with touch screens. Ciman \& Wac [7], for example, analyse the effect of stress on our interaction with a smartphone. They do so based on smartphone gestures analysis (e.g. tap, scroll, swipe, text writing). In [8], on the other hand, the authors try to anticipate and monitor depressive states by monitoring humansmartphone interaction. For this purpose they consider application usage, location and communication logs. This kind of socio-mobile data is used by other authors for similar purposes, such as [9] or [10]. It can also be leveraged to develop more natural and immersive interfaces for Human-Computer Interaction $[11,12]$.

In this paper we follow a different approach to the study of interaction with touch screens. The research cited previously can be characterized as being mostly behavioral, i.e., it is based on the behavior of the user while interacting with the device or its applications and/or while moving about in her/his daily routine. This approach, on the other hand, focuses more acutely on the interaction itself, that is, on the mechanics of the touch. We therefore consider aspects such as the duration of the touch or the variation of its intensity over time. In that sense, this approach is not only behavioral but also physical. Due to its multi-modal nature, we also believe that this approach is better suited to model our interaction with touch screens.

We detail how we build an individual interaction model for each user and how these can be compared. A case study was carried out with 32 participants. The collected data allow us to validate the approach and also show how the participant age significantly influences interaction patterns. We believe that this kind of approach can be useful for better characterizing user interaction, and eventually be applied as a proxy for user state (e.g. stress, emotional state) or user characteristics/traits (e.g. cognitive/physical impairments).

\section{Defining an Interaction Model}

As mentioned in Section 1, in this work we look at the characteristics of each specific touch of the user. Indeed, a touch in the screen of most of nowadays smartphones produces several interaction events throughout the duration of the touch. There is a first event when the finger first touches the screen, which is then followed by several other which are produced as long as the finger is still in 
contact with the screen. Figure 1 depicts an example of a touch, composed by 11 events (red circles) distributed over less than half second. This Figure also shows the shape that is generally associated to a touch: intensity tends to increase and then decrease during the touch.

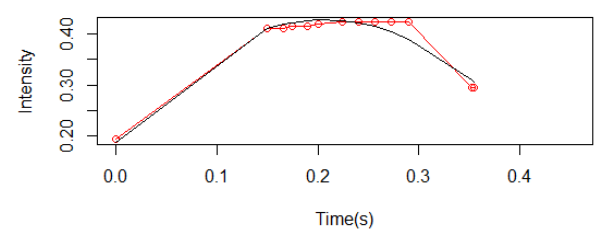

Fig. 1. Intensity of the touch events generated during a touch, over time.

The frequency with which these events are produced is largely dependent on the hardware. Each of these events provides information regarding the intensity of the touch, the area of the finger that is in contact with the screen, the timestamp in which it occurred, etc.

There is thus a significant amount of information that can be used to characterize the interaction of the user. Specifically, the model proposed to characterize user interaction is composed by the following features:

- Touch duration - The duration of each individual touch on the screen;

- Touch intensity - The average, minimum and maximum values of the intensity exerted by the finger on the screen, for each touch;

- Touch area - The average, minimum and maximum values of the area occupied by the finger on the screen, for each touch;

- Intensity values - The sequence of values of intensity generated during each touch, from the moment the finger first touches the screen to the moment it is lifted;

- Area values - The different areas of finger in contact with the screen, in each touch, from the moment the finger first touches the screen to the moment it is lifted;

- Type of action - The actions on the screen can be further characterized (e.g. touch on an active control vs. touch on a layout inactive element);

- Touch pattern - Models how touch intensity varies over time during the touch. Each patient's touch pattern is modeled by fitting a quadratic function to the data (solid black line in Figure 1). The coefficients of the resulting quadratic function are used to characterize the "general shape" of a patient's touch.

As an example, Figure 2 shows the touch patterns of the four different patients, depicted in terms of the intensity values over time and the resulting quadratic function that models it: (a) young male, (b) elder male, (c) young female and (d) elder female. It shows that older users appear to have longer and more intense touches. 
Thus, the interaction model proposed in this work considers a total of 11 features which describe how the user is interacting with the device. They provide new types of information previously not considered in this kind of applications. In this paper we examine these features, namely to find differences in interaction patterns due to socio-demographic variables such as gender and age, although others could also have an influence, such as occupation or health conditions.
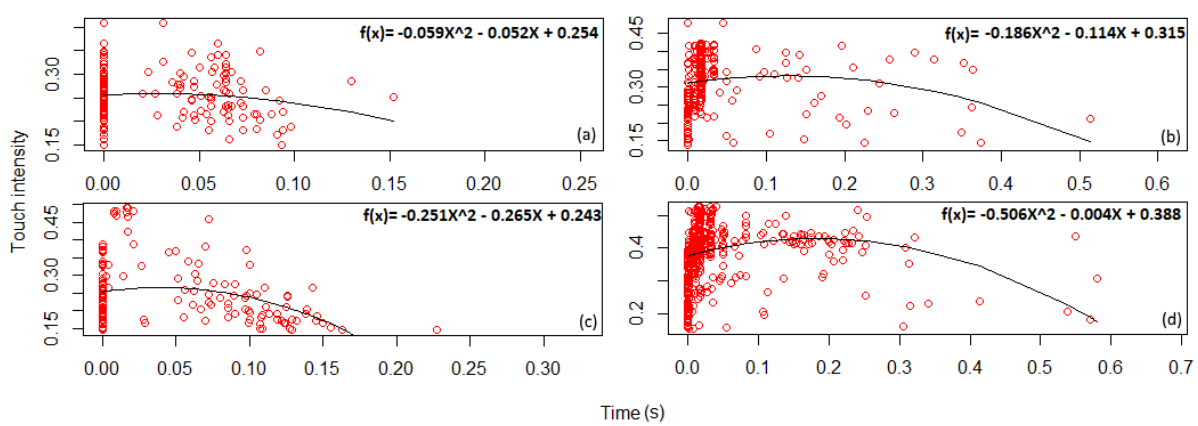

Fig. 2. Interaction models (intensity over time) of four sample users: (a) young male, (b) elder male, (c) young female and (d) elder female.

\section{Case Study}

To assess the validity of the proposed approach and to determine if gender and/or age influence one's interaction with the smartphone, a case study was carried out involving 32 individuals (16 male, 16 female). The average age of the population was $34.91(\min =10, \max =67)$. The distribution of age by gender was also similar: male average age was $35.44(\min =16, \max =67)$ while female average age was $34.38(\min =10, \max =60)$.

The methodology for collecting the interaction data was as follows. A previously developed game-like application for memory stimulation was used. In this application, a new task was created (equal for all participants) that included a memorization task and a recall task. In this application, users explore and navigate a virtual scenario (composed by adjacent still pictures, like in a pointand-click game) while trying to memorize specific aspects. In this case, the virtual scenario comprised 29 still pictures distributed among 2 different virtual rooms. Two specific visual stimuli were added in two pictures, to be used in the recall task. The recall task was administered right after the end of the memorization task, since the goal of this case study is not to study or stimulate memory but rather to evaluate the suitability of the approach to study the users' interaction mechanisms. The recall task was comprised of 7 questions of different types, and used 11 visual stimuli related to the memorization task. 
The users were allowed to interact with the application in a training phase, so that they could get used to the tasks. When ready, the memorization task was started by the researcher, followed by the recall task. Data were collected during both tasks. There was a time limit for the users to complete the memorization task, although there was no minimum time. That is, if the user felt that she/he had already memorized all the necessary detail, they could advance into the recall task. In average, each user spent 5.16 minutes interacting with the application, and touched the screen 79.22 times to complete both tasks, resulting in a dataset with a total of 2535 touches.

\section{Preliminary Data Analysis}

The analysis of the data that is described in this section was carried out with the goal to determine if there are significant interaction differences due to aspects such as age or gender. In the future, we want to carry out similar studies with population with special characteristics, such as mild cognitive impairments. However, at the moment, the goal is to validate the approach.

This section thus details the differences in the aforementioned interaction features when comparing users of different genders or age groups. We focus on the features for which the differences were more significant.

In what concerns the gender, the two variables that better distinguish between male and female users are time between decisions and touch duration. In both cases, female participants tend to exhibit higher values, as Figure 3 shows. The differences observed are statistically significant for both features ( $p$-value $<$ $2.2^{-16}$ and $p$-value $=1.933^{-9}$ ), respectively).
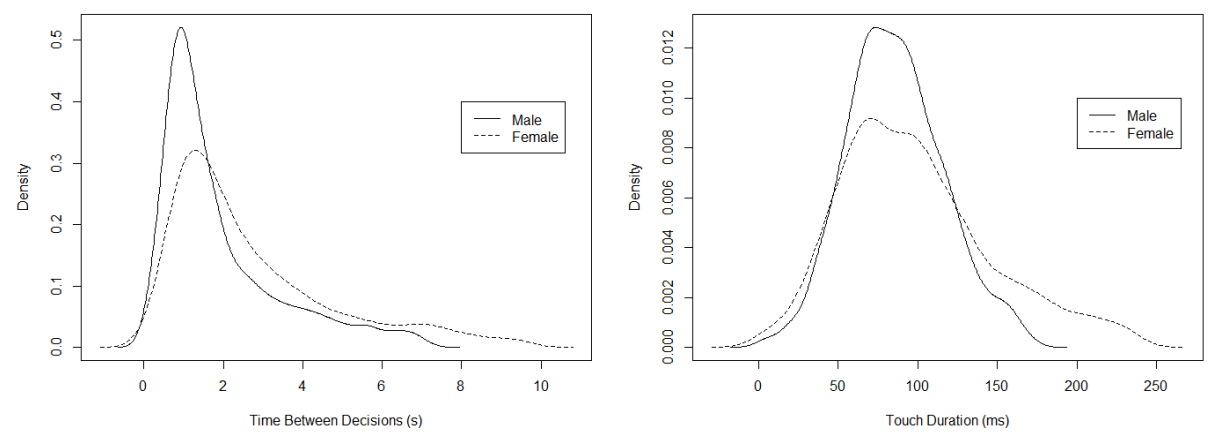

Fig. 3. Gender differences in time between decisions ( $p$-value $\left.<2.2^{-16}\right)$ (left) and in touch duration $\left(p\right.$-value $\left.=1.933^{-9}\right)$ (right) due to gender.

A similar approach was followed to visually and statistically analyze the differences between age groups. For this purpose, participants were grouped according to their age: the so-called young group is composed by users that are 35 
or younger (17 participants), while the old group is constituted by the remaining users (15 participants).

In general, and as expected, older participants tend to have an overall slower interaction, as depicted in Figure 4, which shows the distribution of the data regarding touch duration and time between decisions. Table 1 provides some more detail: touches of older people are, in average, 20.65 milliseconds slower; the time between each two consecutive interactions is also 2.54 seconds slower, in average. The differences between the groups is also statistically significant: $p$-value $=2.909^{-08}$ and $p$-value $=p$-value $=0.025$, respectively.
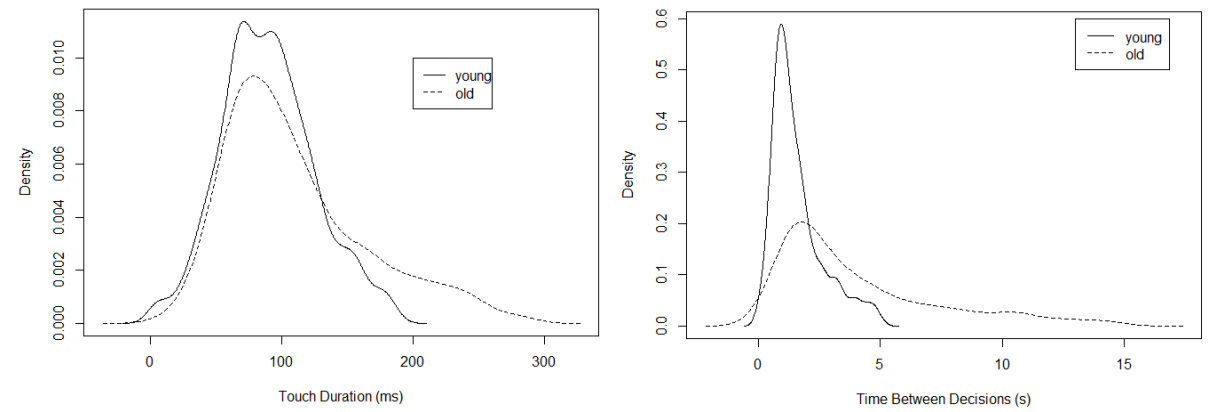

Fig. 4. Differences in touch duration $\left(p\right.$-value $\left.=2.909^{-08}\right)$ (left) and in the time between decisions $(p$-value $=0.025)$ (right) due to age.

Table 1. Summary statistics of the features touch duration (in milliseconds) and time between decisions (in seconds) for both age groups.

\begin{tabular}{cccc|ccc}
\hline \multirow{2}{*}{ Age Group } & \multicolumn{3}{c}{ td } & & \multicolumn{3}{c}{ tbd } \\
& $\bar{x}$ & $\widetilde{x}$ & $\sigma$ & $\bar{x}$ & $\widetilde{x}$ & $\sigma$ \\
\hline Young & 89.45 & 89.45 & 35.36 & 1.58 & 1.26 & 1.06 \\
Old & 110.1 & 97.5 & 54.22 & 4.12 & 2.87 & 3.34 \\
\hline
\end{tabular}

Two other features that are also affected by age, albeit not so significantly, are the average values of touch intensity and touch area during the touch. Figure 5 visually depicts the differences in the distribution of the data. In general, older people tend to have more intense touches and also tend to use a larger area of the finger (average touch area for the older group is 22896 pixels, against 20402 pixels for the younger group). The differences observed are also statistically significant: $p$-value $=2.909^{-08}$ (touch intensity) and $p$-value $=2.643^{-05}$ (touch area).

This section thus shows that both gender and age influence interaction patterns in a significant manner. In section 5 we show that these differences are 

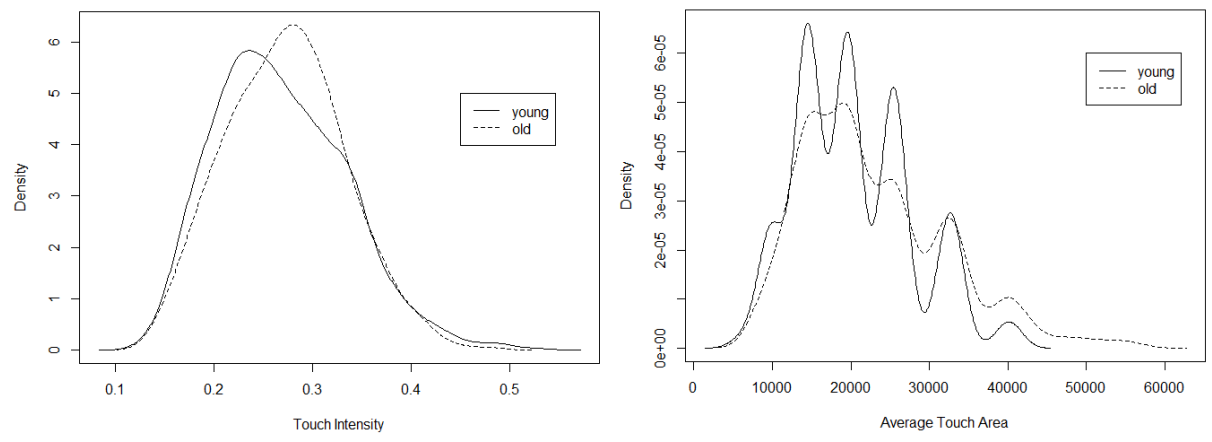

Fig. 5. Differences in the average value of touch intensity $\left(p\right.$-value $\left.=2.909^{-08}\right)$ (left) and in the average value of touch area $\left(p\right.$-value $\left.=2.643^{-05}\right)$ (right) due to age.

enough to train a classifier to distinguish between young and old users with a satisfactory accuracy rate.

\section{$5 \quad$ Results}

After the preliminary analysis of the data described in Section 4, and given the observed differences in terms of interaction in several variables when comparing age groups, work shifted to the train of a model able to distinguish between young and old users. For this purpose, a gradient boosting model was used, based on an ensemble of decision trees. This algorithm is based on an ensemble of weak prediction models (the decision trees), which are gradually improved during training through increasingly refined approximations. In this algorithm, as in other ensembles, predictors are deemed weak in the sense that they are trained on a sample of the instances and/or the variables. For this reason, each predictor is, by itself, a weak one. But the combination of all these predictors generally produces a good model, with a good tendency to generalize. When one of these models is used for classification, as is the case, the output of the model is the most frequent output observed in all the trees.

The dataset used for training the model contains one instance for each touch, and the following 9 variables:

- Maximum, minimum and average touch area - the maximum, minimum and average area of the touch;

- Maximum, minimum and average touch intensity - the maximum, minimum and average intensity of the touch;

- Time between decisions - the time spent since the last touch;

- Touch Duration - the duration of the touch;

- Age bin - a new variable (target variable) added manually, to identify the group age of the user who performed the touch (young or old). 
The trained model (in this case a binomial classification model) is composed of 30 trees, each with a maximum depth of 6 levels. Each of these trees was trained with a random subset of $80 \%$ of the rows of the dataset, and of $70 \%$ of the features.

The model was trained using 5 -fold cross validation. The resulting model correctly classifies $82.03 \%$ of young users and $66.37 \%$ of old users, with an overall $76.08 \%$ of correctly classified instances (precision $=0.6936$, recall $=0.6637, \mathrm{~F} 1$ Score $=0.6783$, AUC $=0.82$ ). Figure 6 shows the plot of the ROC curve (left) and the improvement in the error measure during training.
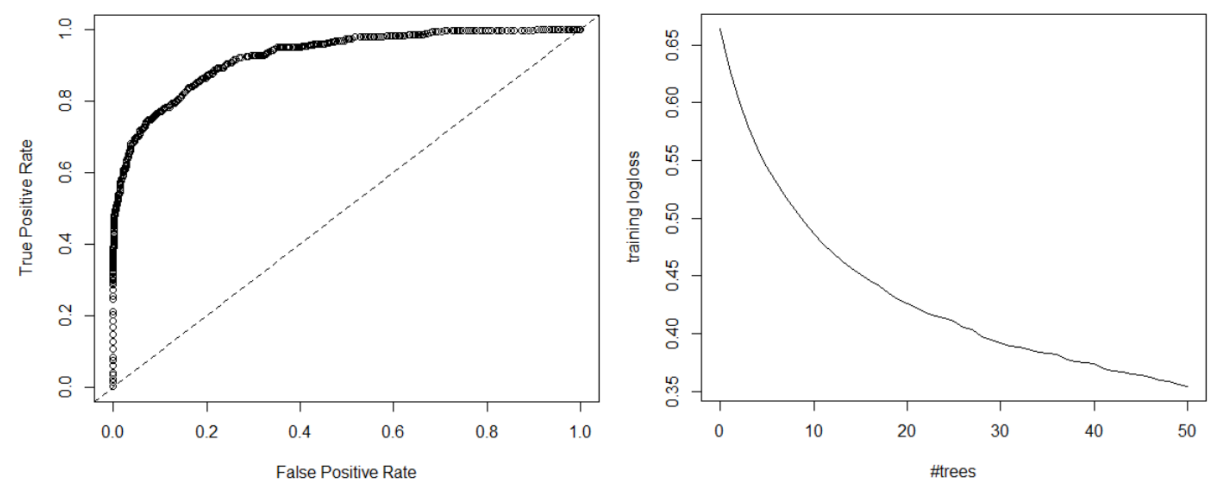

Fig. 6. True positive rate vs. false positive rate $(\mathrm{AUC}=0.82)$ (left); Evolution of error during training (right).

Table 2. Confusion Matrix of the trained model.

\begin{tabular}{cccc}
\hline Actual/Predicted & Young & Old & Error \\
\hline Young & 744 & 163 & 0.1797 \\
Old & 187 & 369 & 0.3363 \\
Total & 931 & 352 & 0.2392 \\
\hline
\end{tabular}

\section{Conclusions and Future Work}

Smartphones and tablets are among the most used technological devices nowadays, which make touch screens a new and privileged form of interaction between humans and technology. In this paper we present a new and multi-modal form of characterizing this interaction, based on behavioral and physical features. Thus, instead of considering previously used features such as applications usage 
patterns, types of gestures or other sensory information (e.g. accelerometer, gyroscope), we use information that characterizes each touch of the user including its intensity, its duration or how these vary over time during the touch.

We conducted a case study in which interaction data was collected from 32 users. The collected data shows that interaction patterns are different between men and women, as well as between people with different age groups. The same data was also used to train a model that is able to distinguish between the user's age group with an accuracy of nearly $74 \%$.

While these results are interesting per se, we believe that this approach can be further pursued not only to study the interaction patterns of large groups of the population (as it allows for an inexpensive process of data collection) but also to study the interaction patterns of people with specific characteristics such as mental or physical disabilities. Indeed, our interaction with these devices is nowadays so pervasive and constant that they are now being used to monitor our health. Interaction patterns may constitute another relevant indicator, namely of the emergence of certain cognitive or physical impairments over time.

We will therefore continue to collect data to widen the population of this study, and include subjects with special characteristics in order to study their specific interaction patterns and thus assess this hypothesis.

\section{Acknowledgments}

This work is co-funded by Fundos Europeus Estruturais e de Investimento (FEEI) through Programa Operacional Regional Norte, in the scope of project NORTE01-0145-FEDER-023577.

\section{References}

1. Oulasvirta, A., Rattenbury, T., Ma, L., Raita, E.: Habits make smartphone use more pervasive. Personal and Ubiquitous Computing 16(1) (2012) 105-114

2. Leeming, K., Swann, W., Coupe, J., Mittler, P.: Non-verbal communication. In: Teaching Language and Communication to the Mentally Handicapped. Routledge (2018) 238-267

3. Carneiro, D., Novais, P., Pêgo, J.M., Sousa, N., Neves, J.: Using mouse dynamics to assess stress during online exams. In: International Conference on Hybrid Artificial Intelligence Systems, Springer (2015) 345-356

4. Pimenta, A., Carneiro, D., Neves, J., Novais, P.: A neural network to classify fatigue from human-computer interaction. Neurocomputing 172 (2016) 413-426

5. Pentel, A.: Predicting age and gender by keystroke dynamics and mouse patterns. In: Adjunct Publication of the 25th Conference on User Modeling, Adaptation and Personalization, ACM (2017) 381-385

6. Nahin, A.N.H., Alam, J.M., Mahmud, H., Hasan, K.: Identifying emotion by keystroke dynamics and text pattern analysis. Behaviour \& Information Technology 33(9) (2014) 987-996

7. Ciman, M., Wac, K.: Individuals' stress assessment using human-smartphone interaction analysis. IEEE Transactions on Affective Computing 9(1) (2018) 51-65 
8. Mehrotra, A., Hendley, R., Musolesi, M.: Towards multi-modal anticipatory monitoring of depressive states through the analysis of human-smartphone interaction. In: Proceedings of the 2016 ACM International Joint Conference on Pervasive and Ubiquitous Computing: Adjunct, ACM (2016) 1132-1138

9. Padmaja, B., Prasad, V.R., Sunitha, K.: Treenet analysis of human stress behavior using socio-mobile data. Journal of Big Data 3(1) (2016) 24

10. Boonstra, T.W., Nicholas, J., Wong, Q.J., Shaw, F., Townsend, S., Christensen, H.: Using mobile phone sensor technology for mental health research: Integrated analysis to identify hidden challenges and potential solutions. Journal of medical Internet research 20(7) (2018)

11. Sanchis, A., Julián, V., Corchado, J.M., Billhardt, H., Carrascosa, C.: Using natural interfaces for human-agent immersion. In: International Conference on Practical Applications of Agents and Multi-Agent Systems, Springer (2014) 358-367

12. Sanchis, Á., Inglada, J., Javier, V., Corchado, J.M., Billhardt, H., Carrascosa Casamayor, C.: Improving human-agent immersion using natural interfaces and cbr. International Journal of Artificial Intelligence 13(1) (2015) 81-93 